\title{
Evaluation of Haemodynamics and Morphology of Carotid Artery in Nonarteritic Anterior Ischaemic Optic Neuropathy
}

Jiali Li ( $\sim$ ljli@mail2.sysu.edu.cn )

Zhujiang Hospital https://orcid.org/0000-0002-9536-9075

\section{Binghao Chen}

Women's and Children's Hospital

Shanshan Liu

Zhujiang Hospital

Ting Chen

Zhujiang Hospital

\section{Research Article}

Keywords: Non-arteritic anterior ischemic optic neuropathy, carotid artery, flow velocity

Posted Date: July 26th, 2021

DOl: https://doi.org/10.21203/rs.3.rs-696920/v1

License: (c) (i) This work is licensed under a Creative Commons Attribution 4.0 International License.

Read Full License 


\section{Abstract \\ Purpose}

To compare haemodynamics and morphological changes of carotid artery in patients with unilateral nonarteritic anterior ischaemic optic neuropathy (NAION) with their contralateral side.

\section{Methods}

Twenty-six patients with unilateral NAION were included in this retrospective study. Colour Doppler imaging (CDI) was used to measure the haemodynamics and morphological changes of carotid artery, including blood flow velocities of common carotid artery (CCA) as well as the diameter of CCA, internal carotid artery (ICA), external carotid artery (ECA) and the thickness of carotid artery plaque. A pairedsample $t$ test was performed to analyze those carotid artery hemodynamics and morphological changes between the affected eye and the unaffected contralateral eye. Subgroup analysis of the blood flow velocities of CCA was performed by dividing patients into two groups according to the presence/ absence of diabetes mellitus, hypertension or hyperlipidaemia.

\section{Results}

The average blood flow velocity of CCA in the affected eyes was $0.45 \pm 0.13 \mathrm{~m} / \mathrm{s}$, greater than that in the unaffected eyes $(0.41 \pm 0.15 \mathrm{~m} / \mathrm{s})(p=0.003 ; \mathrm{t}=-3.321)$. Subgroup analysis found this significant bilateral difference was still existed in groups of diabetes mellitus, non-diabetes mellitus, and hypertension except for groups of non-hypertension, hyperlipidamemia, and non-hyperlipidaemia. The thickness of carotid artery plaque was $0.75 \pm 1.33 \mathrm{~mm}$ in the affected eyes, insignificantly differed from that in the unaffected eyes $(0.55 \pm 0.77 \mathrm{~mm})(p=0.4)$. There were no difference in the diameters of the CCA, ICA, and ECA between the affected and unaffected eyes ( $p=0.862,0.758$, and 0.72 , respectively).

\section{Conclusions}

Increased CCA blood flow velocity might be associated with the development of NAION. Further study on larger scale are needed to confirm our findings.

\section{Introduction}

Acute ischaemia of the optic nerve head is a known cause of non-arteritic anterior ischaemic optic neuropathy (NAION) [1, 2]. There are multiple risk factors for NAION, which are related to hypofusion of the posterior ciliary artery (PCA) circulation $[3,4]$. Some previous studies suggested that haemodynamics and morphological changes, including the velocity of the retrobulbar circulation, and the intima-media thickness (IMT) of carotid artery were associated with the development of NAION. However, the findings 
of these studies were contradictory [5-9]. Some reported decreased circulation velocity of the ophthalmic artery $(O A)$ on the affected side in NAION versus that of the OA on the contralateral unaffected side [6]. However, others found that of OA in the affected side was the same either with unaffected side or the normal controls $[5,7]$. The same situation was also seen in the analysis of the blood flow velocity of the central retinal artery (CRA), with inconsistent results [5, 7]. However, findings on the IMT of common carotid artery (CCA) associated with NAION was commonly consistent, with significant elevation of the IMT in patients with NAION as compared to either contralateral normal side or normal controls [6-9].

To the best of our knowledge, only a few studies have investigated the association of the internal carotid artery (ICA), external carotid artery (ECA), and CCA diameters and CCA blood flow velocity with NAION. According to the Bernoulli's equation, flow velocity increases as a result of vascular constriction or narrowing [10]. The aim of this study was to investigate those carotid artery hemodynamics and morphological changes on affected and unaffected sides in patients with acute unilateral NAION using colour Doppler imaging (CDI).

\section{Methods}

\section{Subjects}

Twenty-six patients with unilateral NAION were included in this retrospective study. Patients were enrolled if they showed acute painless loss of visual acuity, relative afferent pupillary defect, optic disc oedema, visual field defects on automated perimetry, increased retinal arteriovenous passage time, and delayed filling of the disc by fluorescein angiography in the affected eye, without evidence of other neurological, systemic or relevant ocular diseases [2]. The examinations of the non-involved fellow eye were normal in terms of visual acuity, visual field, funduscopy and fluorescein angiography.

Written informed consent was obtained from all the participants or their guardians. The study complied with the tenets of the Declaration of Helsinki.

\section{CDI examinations}

CDI was applied to perform the diameters of the CCA, ECA and ICA, and the blood flow velocity of CCA bilaterally with a high-resolution ultrasound machine equipped with a high-frequency (5-12) MHZ linear array ultrasound probe. Carotid artery plaque was examined using the same ultrasound probe following the European Mannheim consensus [11, 12]. The maximum thickness of the plaques in carotid artery bilaterally was measured. All the measurements were performed by an experienced operator unware of the subjects' condition.

\section{Statistical analysis}

A paired sample $t$ tests was applied for comparisons between the affected and unaffected eyes in the NAION group using SPSS software, version 11.5 (SPSS, Chicago, IL). After normality testing by the Shapiro-Wilk W test, Wilcoxon's test was performed for data with a non-normal distribution. A $p$ value of < 
0.05 was regarded as statistically significant. Subgroup analysis were performed by dividing patients into two groups according to the presence/ absence of diabetes mellitus, hypertension or hyperlipidaemia.

\section{Results}

Of the 26 patients with NAION included in this study, there were 16 were males, with a mean age of $54.4 \pm$ 10.50 years. Among the participants, eight (30.77\%) had a history of diabetes mellitus, eight $(30.77 \%)$ had hypertension, and 11 (42.31\%) had a history of hyperlipidaemia.

The sonographic data on the patients were summarized in Table 1. There were no statistically significant difference in the diameters of the CCA, ICA, and ECA between the affected and unaffected eyes $(p=0.862$, 0.758 , and 0.72 , respectively) (Table 1 ). The thickness of carotid artery plaque was $0.75 \pm 1.33 \mathrm{~mm}$ in the affected eyes, insignificantly differed from that in the unaffected eyes $(0.55 \pm 0.77 \mathrm{~mm})(p=0.4)$ (Table 1). The average blood flow velocity of CCA in the affected eyes was $0.45 \pm 0.13 \mathrm{~m} / \mathrm{s}$, with a maximum velocity of $0.82 \mathrm{~m} / \mathrm{s}$ and a minimum velocity of $0.29 \mathrm{~m} / \mathrm{s}$. The average blood flow velocity of CCA in the unaffected eyes was $0.41 \pm 0.15 \mathrm{~m} / \mathrm{s}$, with a maximum velocity of $0.88 \mathrm{~m} / \mathrm{s}$ and a minimum velocity of $0.23 \mathrm{~m} / \mathrm{s}$. The blood flow velocity of the CCA of the affected eyes were significantly greater than that of the unaffected eyes $(p=0.003 ; \mathrm{t}=-3.321)$.

Subgroup analysis revealed that the blood flow velocities of the CCA were greater in the affected eye than the contralateral eye either in subgroup of diabetes mellitus $(p=0.032)$ or in subgroup of non-diabetes mellitus $(p=0.016)$ (Table 2$)$. A similar finding applied to the subgroup of hypertension $(p=0.014)$ (Table 2). However, there was no difference in the blood flow velocity of the CCA in subgroups of nonhypertension, hyperlipidamemia, and non-hyperlipidaemia between the affected and unaffected eyes ( $p=$ $0.069,0.059$, and 0.054 , respectively) (Table 2).

\section{Discussion}

This retrospective study of 26 patients with unilateral NAION found greater blood flow velocity of CCA in the affected eye than in the contralateral unaffected eye. The same findings were also seen in the subgroup analysis of the presence/ absence of diabetes mellitus and the presence of hypertension except in the subgroup of the absence of hypertension and the presence/ absence of hyperlipidamemia. There were no significant differences in the diameters of the CCA, ICA, ECA and the thickness of carotid artery plaque in the affected eyes versus those if the unaffected eyes.

NAION is a major cause of severe vision impairment or blindness in the middle-aged and elderly population [13]. Despite much study, the pathogenesis of NAION remains unclear, with contradictory findings. The main source of blood supply to the anterior part of the optic nerve is the PCA circulation via the peripapillary choroid and the circle of Zinn and Haller [14]. An ischemic disorder of the PCA circulation in the $\mathrm{ONH}$ is the most frequent cause of NAION [2]. The findings regarding the retrobular haemodynamics associated with $\mathrm{ONH}$ are controversial. We systemically reviewed the literatures on 
retrobular haemodynamics and carotid arteries in patients with NAION and the published results were summarized in Table 3. As shown in Table 3, the findings in the literatures on the blood flow velocity of the OA were inconsistent. Some of them found there was no difference on the blood flow velocity of OA between affected side and the unaffected side[7] or between the affected side and normal controls [5] ,while others found the blood flow velocity of OA were decreased in affected sided compared with the unaffected sided [6]. The findings of the blood flow velocity of CRA also were inconsistent [5, 7]. In individuals with NAION, most published studies reported increased IMT of the CCA on the affected side as compared to that on the unaffected side [6-8], with only one study finding no difference in the IMT of the CCA between the affected and unaffected sides [9].

In our retrospective analysis, we found no significant difference in the diameters of the ICA, ECA and CCA between the affected and unaffected sides of patients $(n=26)$ with NAION. However, the blood flow velocity of CCA in the affected side was increased as compared to that in the unaffected side. Our findings suppport carotid artery blood flow acting like laminar flow of a fluid through a tube, as described by Bernoulli's equation [10], with the flow velocity of the CCA increasing as a result of vascular constriction or narrowing. A previous study detected no significant change in the blood flow velocity of the CCA on the affected side in those with NAION as compared to that in healthy controls [7]. No studies have investigated on comparison of blood flow velocity of the CCA between the affected side and the unaffected side. Based on our findings and those in the literature [5-9], we suppose that the increased blood flow velocity of the CCA might become a marker of NAION prior to changes in the IMT or vacular caliber. Further studies on larger scale are needed to confirm our assumption and to elucidate the normal range of differences in blood flow velocities of CCA between the two sides in a healthy population and in individuals with NAION.

Although we designed a self-control study to exclude potential confounders and enhance the validity of the findings, our study has some limitations. Among potential confounding factors were the differences in the haemodynamics of the affected and unaffected sides. It is possible that there were other unknown differences in the haemodynamics of vessesls of both sides other than that of CCA influenced measurements of the independent variables in the present study. In addition, due to the lack of data from matched normal controls, we were unable to determine whether the range of blood flow velocities or the differences between the affected and unaffected sides were within the range of those observed in a normal population.

In conclusion, increased blood flow velocity of the CCA might be associated with the development of NAION. Increased CCA blood flow velocity might be potentially serve as a marker of the development of NAION prior to other CCA-related abnormalities, such as changes in the IMT or vascular caliber if further study on larger scale is performed in the future.

\section{Declarations}

\section{Funding}


This work was supported by the National Natural Science Foundation of China (Grant numbers 81800871).

\section{Conflicts of interest}

No conflicting relationship exists for any author regarding this manuscript.

\section{Availability of data and material}

Data and material are available from tables.

\section{Code availability}

Not applicable

\section{Authors' contributions}

Jiali Li and Binghao Chen were involved in designing and conducting the study. Jiali Li, Shanshan Liu, and Ting Chen were responsible for data collection and management. Analysis and interpretation of the data were performed by Jiali Li. Jiali Li also prepared the first draft of the manuscript. Jiali Li reviewed and approved the final manuscript. All the authors read and approved the final manuscript.

\section{Ethics approval}

Ethics committee approval was not required for this study, and it adhered to ethical principles outlined in the declaration of Helsinki.

\section{Consent to participate}

Written informed consent was obtained from all the participants or their guardians.

\section{Consent for publication}

All the participants or their guardians agreed to collect and publish the data.

\section{References}

1. Hayreh SS (1997) Anterior ischemic optic neuropathy. Clin Neurosci 4(5):251-263

2. Hayreh SS (2009) Ischemic optic neuropathy. Prog Retin Eye Res 28(1):34-62

3. Biousse V, Newman NJ (2015) Ischemic Optic Neuropathies. N Engl J Med 372(25):2428-2436

4. Rubin DS, Matsumoto MM, Moss HE, Joslin CE, Tung A, Roth S (2017) Ischemic Optic Neuropathy in Cardiac Surgery: Incidence and Risk Factors in the United States from the National Inpatient Sample 1998 to 2013. Anesthesiology 126(5):810-821 
5. Kaup M, Plange N, Arend KO, Remky A (2006) Retrobulbar haemodynamics in non-arteritic anterior ischaemic optic neuropathy. Br J Ophthalmol 90(11):1350-1353

6. Sanjari MS, Falavarjani KG, Mehrabani M, Ghiasian L, Zamani B (2009) Retrobulbar haemodynamics and carotid wall thickness in patients with non-arteritic anterior ischaemic optic neuropathy. $\mathrm{Br} \mathrm{J}$ Ophthalmol 93(5):638-640

7. Zhu W, Cui M, Yao F, Liao R, Liu L (2014) Retrobulbar and common carotid artery haemodynamics and carotid wall thickness in patients with non-arteritic anterior ischaemic optic neuropathy. Graefe's archive for clinical and experimental ophthalmology = Albrecht von Graefes Archiv fur klinische und experimentelle Ophthalmologie 252(7):1141-6

8. Zhu W, Chen T, Jin L, Wang H, Yao F, Wang C et al (2017) Carotid artery intimal medial thickness and carotid artery plaques in hypertensive patients with non-arteritic anterior ischaemic optic neuropathy. Graefe's archive for clinical and experimental ophthalmology = Albrecht von Graefes Archiv fur klinische und experimentelle Ophthalmologie 255(10):2037-43

9. Fu Z, Li H, Wang W, Wang Y (2019) Research on Association of the Diameter of the Internal Carotid Artery Siphon and Nonarteritic Anterior Ischaemic Optic Neuropathy. J Ophthalmol 2019:7910602

10. Hicks JW, Badeer HS (1992) Gravity and the circulation: "open" vs. "closed" systems. Am J Physiol 262(5 Pt 2):R725-R732

11. Chambless LE, Heiss G, Folsom AR, Rosamond W, Szklo M, Sharrett AR et al (1997) Association of coronary heart disease incidence with carotid arterial wall thickness and major risk factors: the Atherosclerosis Risk in Communities (ARIC) Study, 1987-1993. Am J Epidemiol 146(6):483-494

12. Naqvi TZ, Lee MS (2014) Carotid intima-media thickness and plaque in cardiovascular risk assessment. JACC Cardiovascular imaging 7(10):1025-1038

13. Blindness GBD, Vision Impairment C, Vision Loss Expert Group of the Global Burden of Disease $S$ (2021) Causes of blindness and vision impairment in 2020 and trends over 30 years, and prevalence of avoidable blindness in relation to VISION 2020: the Right to Sight: an analysis for the Global Burden of Disease Study. Lancet Glob Health 9(2):e144-e160

14. Bajpai V, Madan S (2021) Arteritic anterior ischaemic optic neuropathy. An update 23:11206721211009447

\section{Tables}

Due to technical limitations, table 1,2,3 is only available as a download in the Supplemental Files section.

\section{Supplementary Files}

This is a list of supplementary files associated with this preprint. Click to download.

- Table1.xlsx

- Table2.xlsx 
- Table3.xIsx

Page $8 / 8$ 